\title{
Evaluation of primary stability of innovated orthodontic miniscrew system (STS): An ex-vivo study
}

\author{
Massoud Seifi ${ }^{1}$, Negin-Sadat Matini ${ }^{2}$ \\ ${ }^{1}$ DDS, MSD, MS Med Edu. Dentofacial Deformities Research Center, Shahid Beheshti University of Medical Sciences, Tehran, \\ Iran \\ ${ }^{2}$ DDS. Department of Orthodontics, Shahid Beheshti University of Medical Sciences, Tehran, Iran
}

Correspondence:

Daneshjoo Blvd

Dental School-Orthodontic Department

Evin, Tehran, Iran. 19839-69411

negin.matini@hotmail.com

\begin{abstract}
Seifi M, Matini NS. Evaluation of primary stability of innovated orthodontic miniscrew system (STS): An ex-vivo study. J Clin Exp Dent. 2016;8(3):e255-9.

http://www.medicinaoral.com/odo/volumenes/v8i3/jcedv8i3p255.pdf
\end{abstract}

Received: 19/07/2015 Accepted: 12/03/2016

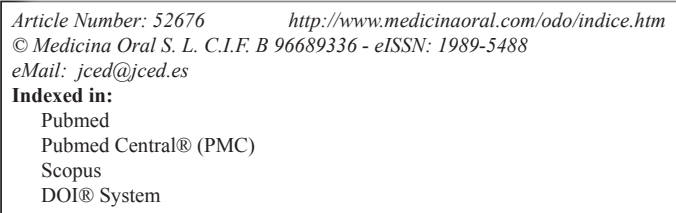

\begin{abstract}
Background: Stability is determined as one of the requirements in use of Temporary Anchorage Devices (TAD) in orthodontics. Miniscrew has been a widely used Bone Anchor. Compared with mini-implant that necessitates osseointegration; mechanical retention is a determining factor for primary stability of miniscrew. Studies investigated various ways to increase primary stability. The aim of this study is to introduce a new configuration of miniscrew system which is believed to obtain more primary stability.

Material and Methods: Freshly ovine mandibles were cut in blocks. Twenty-seven miniscrews (diameter $1.6 \times 8$ mm; G2, Dual Top Anchor System, Jeil Medical, Seoul, Korea) were inserted in the blocks and divided in 2 experimental groups: single miniscrew and the innovated design "Seifi Twin Screw (STS)". Primary stability was evaluated by Periotest "M" $®$ device.

Results: Independent t-test showed a significant difference between 2 experimental groups in periotest evaluation $(p<0.05)$. STS demonstrated higher primary stability due to its mechanical configuration and design.

Conclusions: The STS provides higher primary stability and was found to be effective in increased success rate of miniscrew systems from the standpoint of primary stability.
\end{abstract}

Key words: Anchorage procedures, anchorage techniques, orthodontic anchorage procedures, miniscrews, temporary anchorage device.

\section{Introduction}

Stable Anchorage is one of the major factor in successful orthodontic treatment. Skeletal anchorage is used as one of the temporary anchorage devices (TAD), especially in complicated cases. Miniscrews are examples for skeletal anchorage which are used widely in different sites of mandible and maxilla $(1,2)$. They reduce the need for dental anchorage and can provide different tooth movements without patient's cooperation. There are other advantages of miniscrews as TADs such as non-invasive insertion procedure, providing rigid anchorage against orthodontic loads and minimal anatomic limitation for placement $(2,3)$. However, there are still problems which have effects on the success rate of miniscrew-as- 
sisted treatments. Because of immediate loading on orthodontic miniscrews, primary stability became a basic requirement for loading forces on miniscrews $(4,5)$. It is considered as clinical condition of miniscrew immobility and capacity to withstand loads in different directions (6). The primary stability of miniscrews is mostly supported by mechanical retention between bone and miniscrew surface $(7,8)$. Primary stability is influenced by factors such as overloading (5), bone density (6,9-11), cortical bone thickness $(12)$, screw design $(13,14)$ and root proximity (15).

Studies about different variety of miniscrew designs to improve primary stability are increasing. Different changes in screw diameter, length and the design of the threads have been investigated (16).

There are different methods to assess miniscrew primary stability. Measuring insertion torque, resonance frequency analysis (RFA) and periotest value (PTV). The force used to insert the implant is called insertion torque (17), insertion torque is related to bone tissue, cortical bone thickness and bone density. Adequate insertion torque is an indicator of mini implant stability (14). It should be as high to ensure stability and as low enough to prevent overcompression of the bone. Resonance frequency analysis is also another method for quantitative measurement of primary stability, RFA value is assessed by attaching a transducer directly to the implant (18). In this device, a magnetic piece called "SmartPeg" is screwed on top of the implant head. A handpiece emits electromagnetic impulses to SmartPeg in order to detect the resonance frequency of SmartPeg implant unit (4). A noninvasive device called periotest is used for analysis of implant stability. This device originally developed to measure damping effect of periodontal ligament around natural tooth. The range of PTV depends on damping characteristic of periodontal ligament around tooth (13). It can also assess the mobility of implants and it has been used to measure primary stability of orthodontic miniscrews. The periotest device (Medzintechnik Gulden, Modautal, Germany), produces a transient vibration by tapping the implant as a rod inside the periotest handpiece which is electromagnetically accelerated. The device shows a quantitative reading from -8 (clinically rigid) to +50 (very mobile) (19). More negative PTV means more stability of the implant. Wireless Periotest device (Periotest " $\mathrm{M}$ ") is the recent design introduced for measuring stability in implant and orthodontic miniscrew. It is easy to use in clinical approach and shows reasonable and reproducible results from implant-bone interface (19).

All methods above have been evaluated in different studies. RFA and PTV are noninvasive measurements for stability and they have shown reliability and sensitivity (18).

We designed an innovated system, Seifi Twin Screws (STS), for skeletal anchorage by coupled miniscrews.
The system has been used for force eruption and extrusion of impacted canine by the author. The author suggests that this system demonstrates more primary stability compared with previous designs.

The aim of our study is to introduce the innovated STS and evaluate the primary stability of it compared with conventional single miniscrew anchorage system by PTV measurements.

\section{Material and Methods}

-Specimen Preparation

Freshly ovine mandibles were cut into $10 \mathrm{~cm}$ long pieces under profuse saline-solution cooling (legal permission was obtained from Institutional Review Board). A total of 18 bone blocks were prepared after removing soft tissue. To determine cortical bone thickness and trabecular bone density, each bone block was scanned by Cone Beam Computed Tomography (CBCT) unit (NewTomVGi, Verona, Italy). The radiation exposure was set on High Resolution with $6 \mathrm{~cm} \times 6 \mathrm{~cm}$ Field Of View (FOV). In order to equalize specimens, areas of similar cortical bone thickness and trabeculation were assessed as the insertion site of miniscrews for each bone block. The assessment of the scans was performed by the manufacturer recommended software (NNT Viewer). Then, bone blocks were allocated in two groups of single miniscrew and the innovated system (STS), each group contained 9 blocks.

-Experimental Groups and Configuration of STS

Twenty-seven self-drilling orthodontic miniscrews (diameter $1.6 \times 8$ mm; G2, Dual Top Anchor System, Jeil Medical, Seoul, Korea) were used. The insertion of all miniscrews were done by a single operator. For single miniscrew group, one miniscrew was inserted perpendicular to the bone surface of each block assisted by handheld screw driver (Dual Top Anchor System, Jeil Medical, Seoul, Korea) according to the manufacturer. All miniscrews were placed with no pilot hole. For group two (STS), one miniscrew was first inserted to block; then, the second miniscrew was inserted parallel to the first one with the distance that the tissue suppression stops of both miniscrews were in contact at the proximal aspect. To complete the configuration of the STS, an 18" $\times 25$ " stainless steel rectangular wire (Dentaurum, Ispringen, Deutschland), passed through both miniscrews slots; this wire act as horizontal retentive arm and were fixed by ligature wires (Dentaurum, Ispringen, Deutschland) which were engaged in eyelets that were placed at the neck of the miniscrews (Schematic configuration of STS is illustrated in figure 1).

-Primary Stability measurements

The primary stability measurement was conducted using the periotest " $M$ " $\circledR$ device (Medzintechnik Gulden, Modautal, Germany). According to the manufacturer, the tip of the periotest was placed perpendicular to the minis- 


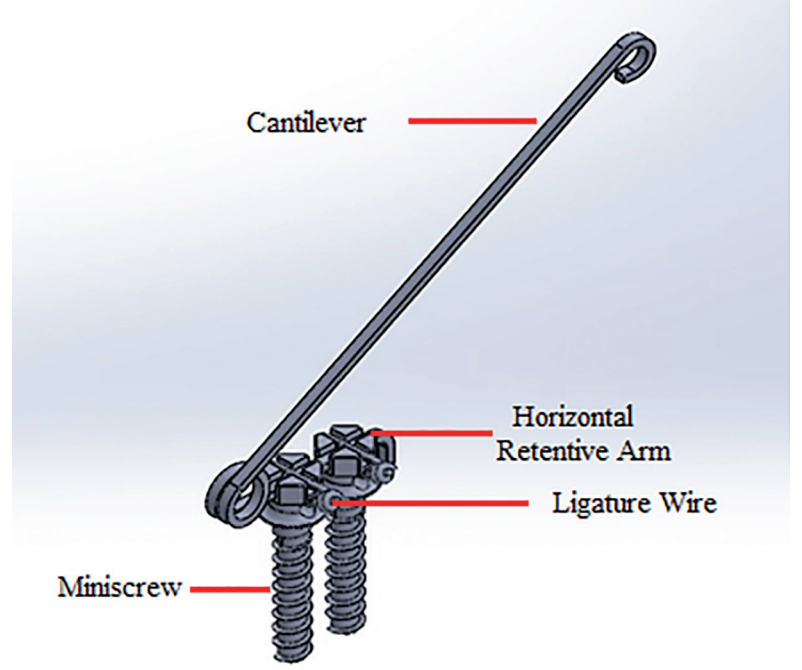

Fig. 1. Seifi Twin Screw (STS): Schematic view.

crew and was held approximately $2 \mathrm{~mm}$ away from the miniscrew head. This device measures the time that the rod remains in contact with the miniscrew; shorter contact time indicates more stability of miniscrew. Values were detected three times for each sample and entered to Excel 2013 for further analysis. Because of the contact between tissue platforms and the wire, the STS was considered as a single unit; so, based on the pilot study which showed no significant difference in PTV between two miniscrews in STS; the periotest measurements were only performed for one of the miniscrews for each system (Fig. 2).

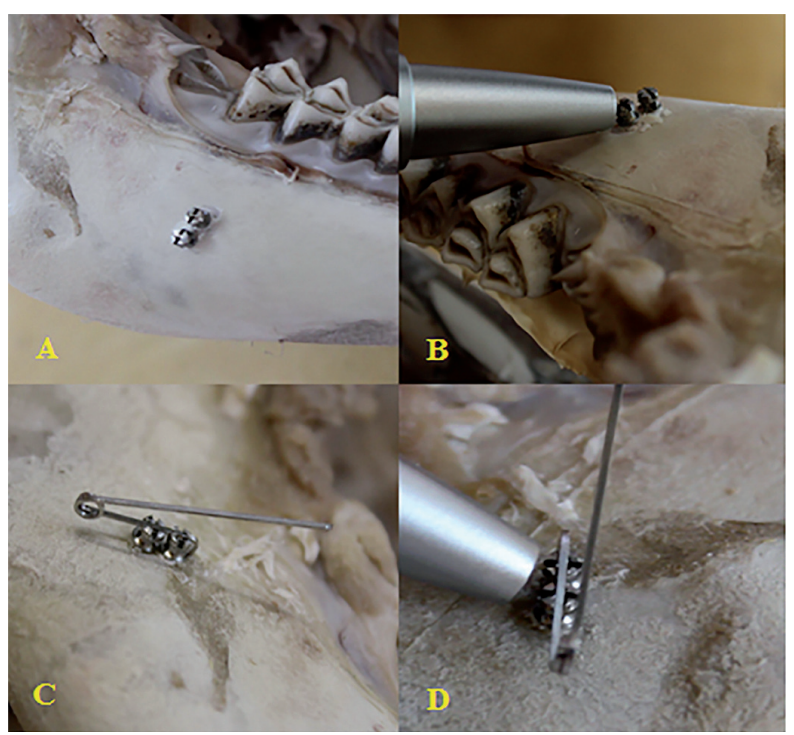

Fig. 2. A) Insertion of both miniscrews in bone block. B) Periotest evaluation on a single screw. C) STS configuration is completed and the wire is in place. D) Evaluation of periotest for STS.
-Statistical Analysis

Data were tested for normal distribution by KolmogorovSmirnov test. The independent t-test was performed for comparison of PTV between two experimental groups using statistical software SPSS (Statistical Package for the Social Sciences, New York, USA) version 21.

\section{Results}

The mean (SD) values of PTV in single screw and STS groups are displayed in table 1. The Kolmogorov-Smirnov test showed normal distribution for the PTV values in both experimental groups $(p=0.2)$. The independent ttest revealed significant difference between single screw and STS groups for PTV. The mean value of PTV in the innovated system was significantly higher than the single screw system $(p=0.025)$.

Table 1. Evaluation of PTV in test groups.

\begin{tabular}{|l|c|c|}
\hline \multirow{2}{*}{ Test groups } & \multicolumn{2}{|c|}{ periotest value (PTV) } \\
\cline { 2 - 3 } & Mean (SD) & $\boldsymbol{p}$-value \\
\hline STS & -5.7032 & \\
\hline Single & -4.3540 & 0.025 \\
\hline
\end{tabular}

Based on results regarding increased stability in STS. A force distribution can be analyzed and compared between single screw system and STS. As demonstrated in figure 3. In STS, as we applied periotest's rod perpendicular to the long axis of miniscrew; the horizontal retentive arm between screws transfers the force to the other miniscrew and it resists against displacement and caries out a part of the force. The maximum tension probably is decreased and increased stability results reduced micro movements of miniscrews due to periotest evaluation. In the other hand, when we applied force to the single miniscrew system, statically, the force is resisted by a triangular distribution around the center of rotation. In application of single miniscrew, maximized reaction is produced in top and bottom of the miniscrew body, which produces excessive tension to the surrounded bone and reduced stability.
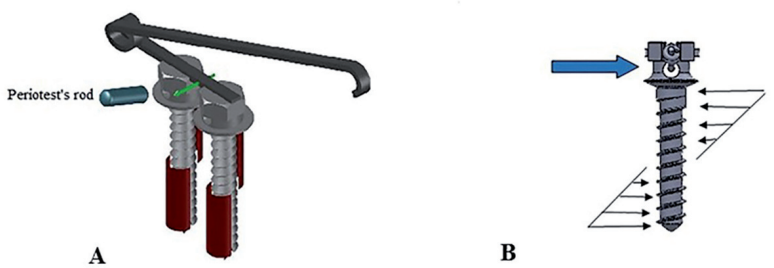

Fig. 3. A) Periotest Evaluation on STS. B) Force distribution by applying periotest's rod on a single miniscrew. 


\section{Discussion}

Stability has a notable effect in achieving successful skeletal anchorage. Studies demonstrated different factors that have correlation with stability of orthodontic miniscrews $(20,21)$. Because of increasing the need for immediate loading, primary stability is the utmost importance (22). The stability should be checked immediately after the insertion of miniscrew, any evidence of miniscrew loosening within the bone results failure of orthodontic treatment in nearly future (20). Primary stability depends on the mechanical engagement of miniscrew and bone; hence, it does not require a period for osseointegration (17). Lack of primary stability can lead to mobility of the miniscrew and subsequently failure of the treatment (23). Bone quality and quantity at the receptor site $(11,12)$, design of the miniscrew (10) and insertion technique (24) are the factors to be considered to ensure primary stability. Due to the lack of clinicians control over bone quality and quantity, miniscrew design and insertion techniques can be the variables in order to enhance success of the procedure. Self-drilling miniscrews doesn't require predrilling of the bone prior the miniscrew insertion. This technique improves the mechanical interlock between bone and the device. Several studies reported wider and longer miniscrews in spite of increase bone-screw interface and contact area (25). A study by Nienkemper et al. (26) described higher stability of $11 \mathrm{~mm}$ mini-implants in initial insertion at midpalatal region compared with $9 \mathrm{~mm}$ mini-implants. In contrast to the mentioned study (26), $8 \mathrm{~mm}$ miniscrews were used for STS and it showed increased primary stability in consistent with previous retrospective studies (27).

The area of miniscrew insertion is also concerned. Many investigators believe that the maxilla has more success rate for miniscrews compared to mandible. The palatal area is superior option for miniscrews insertion and the first author include midpalatal area for the insertion of STS.

Without any change to the shape of miniscrews, we have introduced a novel designed system for skeletal anchorage i.e. two orthodontic self-drilling miniscrews (diameter $1.6 \times 8 \mathrm{~mm}$ ). The advantages of self-drilling miniscrews compared with self-tapping are less operation time, less bone debris, decreasing thermal damage and also patient's comfort (5). Both miniscrews were connected by a rectangular wire which passes through both miniscrew slots and acts as a horizontal retentive arm. The wire was locked in the slots by ligature wires that were tied through built-in eyelets on the neck of each miniscrew. Based on our results, this system (STS), improves primary stability in comparison to the single miniscrew anchorage. The above mentioned design i.e. STS has increased contact surface with the bone. The horizontal retentive arm that has linked both miniscrews to each other creates a truss. The "truss effect" organized two components; makes both miniscrews behave as a single object. The Truss creates shearing force between two miniscrews in eccentricity. It resist distortion from applied forces in any direction and fastened the system in all degrees of freedom. Authors believe that STS is more stable than single miniscrew because it can resists counteracting moments and forces in $\mathrm{X}, \mathrm{Y}$, and $\mathrm{Z}$ axes. Periotest " $M$ " $~($ device is a reliable indicator for measurement of implants stability in both conventional and immediate loadings. This device has a high capacity regarding the determination of miniscrew stability/looseness in in-vitro studies. The PTV quantitative results from STS showed more stability compared with single miniscrew $(p<0.05)$. In other words, STS is able to reduce the amount of time which the rod of the periotest is in contact with the miniscrew (the technology which is used for quantitative assay).

The present study represents an innovated design for skeletal anchorage devices without any intervention to the miniscrew designed by the manufacturer. Tozlu et al. (28), has created an apparatus (a miniscrew ring) which was placed at the neck of the screw. The mentioned study claimed that this ring is able to increase stability due to increasing surface contact of bone with miniscrew. It also has spines which resist from the loading forces; punching the tissue is required to insert spines. Our study is based on the effect of a truss support to prevent moments and forces which cause rotation of miniscrews in bone and eventually miniscrew mobility after the application of load. STS does not require tissue punch or any additional manufactured apparatus and the configuration can be done by conventional orthodontic appliances (miniscrew, rectangular wire, ligature wire).

Youn et al. (7), demonstrated the stability of Hollow type miniscrew compared with C-type miniscrew in beagle dogs. The newly designed H-type miniscrew has 4 fenestration holes for ingrowth of bone. Results concluded that the use of H-type depends on local bone quality and it can be used in maxilla and the C-type in mandible. Also, the fenestration holes in H-type miniscrew is designed to increase osseointegration and it does not have an effect on primary stability. STS is made by miniscrews that can be inserted in most areas of maxilla and mandible. This system has increased surface contact of bone at the insertion site which improves mechanical interlocking of bone and miniscrew.

In clinical approach, anatomical consideration is another limitation for miniscrew insertion. Authors suggest midpalatal area for the insertion of STS which has reduced risk of root damage and has enough bone structure for insertion of miniscrews as can be evaluated in coronal sections of CBCT's. Studies claimed that tongue irritation may occurred when miniscrew is placed in palatal area. Applying bonding resin and composites (flow-able 
or regular body) on the miniscrew heads in STS creates a smooth surface and diminish plaque accumulation.

Histologic evaluation and tissue response to the miniscrews have a bulk of literature support $(17,24)$ but the present study was conducted on the ovine bone blocks for studying the mechanics of the forces and moments applied and the counteracting elements of the system. Clinical cases will determine the feasibility of this system in practice and adequate data regarding the efficacy of the STS will be published in near future in conjunction with the available data. Further studies are needed to investigate stability overtime by applying different types of force on STS.

\section{Conclusions}

The innovated system of STS, has showed increased primary stability compared to a single miniscrew. The STS configuration can distribute the impacted energy in a larger area and in various orientations for counteracting unwanted dislodging forces or moments. The quantitative evaluations suggest that STS can be used as an advantageous skeletal anchorage device in orthodontic treatments.

\section{References}

1. Kim YH, Yang SM, Kim S, Lee JY, Kim KE, Gianelly AA, et al. Midpalatal miniscrews for orthodontic anchorage: factors affecting clinical success. Am J Orthod Dentofacial Orthop. 2010;137:66-72.

2. Kuroda S, Sugawara Y, Deguchi T, Kyung HM, Takano-Yamamoto T. Clinical use of miniscrew implants as orthodontic anchorage: success rates and postoperative discomfort. Am J Orthod Dentofacial Orthop. 2007;131:9-15.

3. Crismani AG, Bertl MH, Celar AG, Bantleon HP, Burstone CJ. Miniscrews in orthodontic treatment: review and analysis of published clinical trials. Am J Orthod Dentofacial Orthop. 2010;137:108-13.

4. Nienkemper M, Wilmes B, Panayotidis A, Pauls A, Golubovic V, Schwarz F, et al. Measurement of mini-implant stability using resonance frequency analysis. Angle Orthod. 2013;83:230-8.

5. Son S, Motoyoshi M, Uchida Y, Shimizu N. Comparative study of the primary stability of self-drilling and self-tapping orthodontic miniscrews. Am J Orthod Dentofacial Orthop. 2014;145:480-5.

6. Oh JS, Kim SG. Clinical study of the relationship between implant stability measurements using Periotest and Osstell mentor and bone quality assessment. Oral Surg Oral Med Oral Pathol Oral Radiol. 2012;113:e35-40.

7. Youn JW, Cha JY, Yu HS, Hwang CJ. Biologic evaluation of a hollow-type miniscrew implant: an experimental study in beagles. Am J Orthod Dentofacial Orthop. 2014;145:626-37.

8. Park HS, Jeong SH, Kwon OW. Factors affecting the clinical success of screw implants used as orthodontic anchorage. Am J Orthod Dentofacial Orthop. 2006;130:18-25.

9. Marquezan M, Osório A, Sant'Anna E, Souza MM, Maia L. Does bone mineral density influence the primary stability of dental implants? A systematic review. Clin Oral Implants Res. 2012;23:767-74.

10. Migliorati M, Benedicenti S, Signori A, Drago S, Barberis F, Tournier $\mathrm{H}$, et al. Miniscrew design and bone characteristics: an experimental study of primary stability. Am J Orthod Dentofacial Orthop. 2012;142:228-34.

11. Samrit V, Kharbanda OP, Duggal R, Seith A, Malhotra V. Bone density and miniscrew stability in orthodontic patients. Aust Orthod J. 2012;28:204-12.

12. Marquezan M, Mattos CT, Sant'Anna EF, de Souza MM, Maia LC. Does cortical thickness influence the primary stability of miniscrews?:
A systematic review and meta-analysis. Angle Orthod. 2014;84:1093103.

13. Maria O, Ana M, Andreu P. Primary stability of microscrews based on their diameter, length, shape and area of insertion. An experimental study with Periotest. Prog Orthod. 2008;9:82-8.

14. Alrbata RH, Ha DW, Yu W, Kyung HM. Optimal asymmetric thread for orthodontic microimplants: Laboratory and clinical evaluation. Angle Orthod. 2015;85:585-90.

15. Shigeeda T. Root proximity and stability of orthodontic anchor screws. J Oral Sci. 2014;56:59-65.

16. Kim YK, Kim YJ, Yun PY, Kim JW. Effects of the taper shape, dual-thread, and length on the mechanical properties of mini-implants. Angle Orthod. 2009;79:908-14.

17. Cehreli S, Arman-Ozcirpici A. Primary stability and histomorphometric bone-implant contact of self-drilling and self-tapping orthodontic microimplants. Am J Orthod Dentofacial Orthop. 2012;141:18795.

18. Al-Jetaily S, Al-Dosari AA. Assessment of Osstell TM and Periotest ${ }^{\circledR}$ systems in measuring dental implant stability (in vitro study). Saudi Dent J. 2011;23:17-21.

19. Crum PM, Morris HF, Winkler S, DesRosiers D, Yoshino D. Wi$\mathrm{red} /$ Classic and Wireless/Periotest " $\mathrm{M}$ " instruments: an in vitro assessment of repeatability of stability measurements. J Oral Implantol. 2014;40:15-8

20. Wilmes B, Rademacher C, Olthoff G, Drescher D. Parameters affecting primary stability of orthodontic mini-implants. J Orofac Orthop. 2006;67:162-74.

21. Manni A, Cozzani M, Tamborrino F, De Rinaldis S, Menini A. Factors influencing the stability of miniscrews. A retrospective study on 300 miniscrews. Eur J Orthod. 2011;33:388-95.

22. Romanos GE. Bone quality and the immediate loading of implantscritical aspects based on literature, research, and clinical experience. Implant Dent. 2009;18:203-9.

23. Romanos GE, Toh CG, Siar CH, Swaminathan D. Histologic and histomorphometric evaluation of peri-implant bone subjected to immediate loading: an experimental study with Macaca fascicularis. Int J Oral Maxillofac Implants. 2002;17:44-51.

24. Chen Y, Shin HI, Kyung HM. Biomechanical and histological comparison of self-drilling and self-tapping orthodontic microimplants in dogs. Am J Orthod Dentofacial Orthop. 2008;133:44-50.

25. Lim SA, Cha JY, Hwang CJ. Insertion torque of orthodontic miniscrews according to changes in shape, diameter and length. Angle Orthod. 2008;78:234-40.

26. Nienkemper M, Wilmes B, Pauls A, Drescher D. Impact of miniimplant length on stability at the initial healing period: a controlled clinical study. Head Face Med. 2013;9:30.

27. Lim HJ, Eun CS, Cho JH, Lee KH, Hwang HS. Factors associated with initial stability of miniscrews for orthodontic treatment. Am J Orthod Dentofacial Orthop. 2009;136:236-42.

28. Tozlu M, Nalbantgil D, Ozdemir F. Effects of a newly designed apparatus on orthodontic skeletal anchorage. Eur J Dent. 2013;7:S83-8

\section{Acknowledgements}

We would like to thank Ali Karkhaneh, School of Mechanical Engineering, Faculty of Engineering, University of Tehran, Tehran, Iran; for creating graphic images. The present article is based on the theological part of the dissertation by Dr. Matini under supervision of Prof. Seifi.

\section{Conflict of Interest}

The authors declare that they have no conflict of interest. 